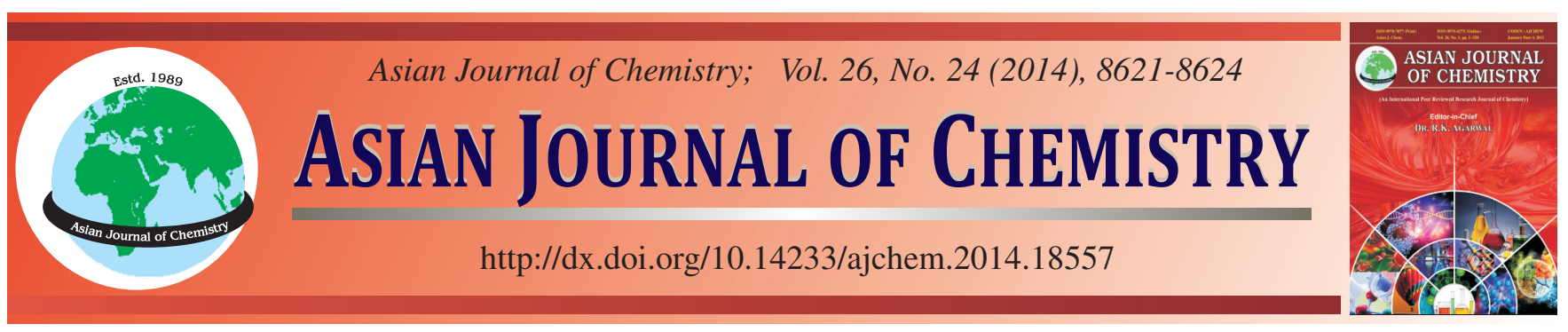

\title{
Development of Method to Determine C6-C3 Phenolic Acids in Tobacco (Nicotiana tabacum) by HPLC
}

\author{
Huina Zhao, Kai Cai, Bo Lei ${ }^{*}$, Zhu Ren, Bin Cai and Wenje Pan ${ }^{*}$
}

Guizhou Academy of Tobacco Science, Guiyang 550081, Guizhou Province, P.R. China

*Corresponding authors: Fax: +86 851 4116909; Tel: +86 18985590078; E-mail: leibo_1981@163.com; wenjiepan@163.com

\begin{abstract}
A method to simultaneous determination of four C6-C3 phenolic acids in tobacco by high performance liquid chromatography (HPLC) was established. Four C6-C3 phenolic acids were well separated in $40 \mathrm{~min}$. The proposed method had high sensitivity, accuracy and repeatability with good linear relationship $\left(\mathrm{R}^{2} \geq 0.9992\right)$ when the concentration of phenolic acids was between 1.113 and 102.332 $\mu \mathrm{g} / \mathrm{mL}$. This method was applied to tobacco samples. The results showed that the content of four C6-C3 phenolic acids was significantly different in tobacco at different regions and growth stages, and the contents of $p$-coumaric acid, ferulic acid and sinapic acid in tobacco were influenced by the ecological conditions more than growth stages. This might be one of the reasons that the tobacco leaves in different regions had different quality.
\end{abstract}

Keywords: Tobacco, HPLC, C6-C3 phenolic acids.

\section{INTRODUCTION}

In recent years, phenolic acids have attracted widespread attention as potential antioxidant activity, anticancer effects, healthcare functions for chronic diseases and their ubiquity in a wide range ${ }^{1-4}$. There are two types of main skeleton of phenolic compounds found in nature: (1) C6-C1 phenolic compounds. The basic skeleton is benzoic acid, for example, protocatechuic acid, vanillic acid, syringic acid, gallic acid, etc. (2) C6-C3 phenolic compounds. They are mainly present in the form of cinnamic acid derivatives, such as caffeic acid, ferulic acid, sinapic acid and $p$-coumaric acid, etc. It usually exists in the form of conjugates which connected together with quinic acid or glucose $\mathrm{e}^{5}$.

The C6-C3 phenolic compounds which comprised of phenolic hydroxyl and carboxyl compounds were the intermediate products of phenylpropanoid metabolic pathway and the metabolite of lignin biosynthesis in tobacco. C6-C3 phenolic compounds were closely related to adversity resistance and physiological activity of tobacco. Therefore, the determination of C6-C3 phenolic acids in tobacco is important for judging the quality and flavor of tobacco.

Determination of phenolic compounds by high performance liquid chromatography (HPLC) has been reported in plant, such as fruits ${ }^{6,7}$, vegetables ${ }^{8,9}$, honey ${ }^{10-12}$, medicinal plants ${ }^{13}$, food crops ${ }^{14,15}$ and herbs ${ }^{16}$. However, it took 40-50 min or even 70-90 min to separate the main phenolic compounds including $p$-coumaric acid, sinapic acid and ferulic acid with poor separation effects ${ }^{7,18,19}$. To the best of our knowledge, there were no systematic reports in the literature regarding the determination of C6-C3 phenolic compounds ( $p$-coumaric acid, ferulic acid and sinapic acid) in tobacco. In the present study, we set out to investigate the method for determination of the C6-C3 phenolic compounds in tobacco with a short analysis time by HPLC.

\section{EXPERIMENTAL}

The reference substances including caffeic acid, $p$-coumaric acid, ferulic acid and sinapic acid were reagent grade with purity $\geq 98 \%$ and purchased from Sigma-Aldrich (St. Louis, MO, USA). The internal substance cinnamic acid was reagent grade with purity $\geq 98 \%$ and purchased from Beijing Century Aoke Biotechnology Reagent. (Beijing, China). Acetonitrile, acetic acid and other reagents were HPLC grade and purchased from Tedia Co. (Tedia, USA).

Collection and pretreatment of tobacco sample: The 11th leaves (from the bottom to top) of tobacco (Nicotiana tabacum L. K 326) were collected from five regions (Heilongjiang, Shanxi, Shandong, Guizhou and Chongqing) at five growth stages (every 10 days after flowering until one day before harvest), respectively. Immediately after the sampling, the leaves were frozen with liquid nitrogen. Then all samples were dried with vacuum freeze-drying until constant weight (no water) and ground to powder for the analysis of C6-C3 phenolic compounds. 
Free phenolic acids extraction: Accurately $0.6 \mathrm{~g}$ of tobacco sample was weighted into a flask and $200 \mu \mathrm{L}$ of $100 \mu \mathrm{g} / \mathrm{mL}$ cinnamic acid (internal standard) was also added. $20 \mathrm{~mL}$ extraction solvent were added and then ultrasound extraction was used $(100 \mathrm{~Hz}$, room temperature). At last, the extraction solution was filtered with $0.22 \mu$ m nylon membrane. The different extraction reagent and time were as shown in Table-1.

TABLE-1

EXTRACTION CONDITIONS OF FREE PHENOLIC ACIDS

Extraction solvent Ultrasound extraction time (h)

\begin{tabular}{cc} 
Extraction solvent & Ultrasound extraction time $(\mathrm{h})$ \\
\hline \multirow{2}{*}{$70 \%$ methanol } & 0.5 \\
& 1.0 \\
& 2.0 \\
\hline \multirow{2}{*}{$80 \%$ methanol } & 0.5 \\
& 1.0 \\
& 2.0 \\
\hline \multirow{2}{*}{$100 \%$ methanol } & 0.5 \\
& 1.0 \\
& 2.0 \\
\hline
\end{tabular}

Bound phenolic acids extraction: Accurately $0.6 \mathrm{~g}$ of tobacco sample was weighted into a flask and $200 \mu \mathrm{L}$ of 100 $\mu \mathrm{g} / \mathrm{mL}$ cinnamic acid (internal standard) was also added. The sample was hydrolyzed by $40 \mathrm{~mL}$ of different concentration of $\mathrm{NaOH}$ solutions $(1,2$ or $4 \mathrm{~mol} / \mathrm{L})$ with $4 \mathrm{~h}$ oscillation and then centrifuged $15 \mathrm{~min}$ at $4{ }^{\circ} \mathrm{C}$ with $10000 \mathrm{rpm}$. The supernatant was transferred into another flask and the $\mathrm{pH}$ was adjusted to 2 using $6 \mathrm{~mol} / \mathrm{L} \mathrm{HCl}$. After extracted three times with $20 \mathrm{~mL}$ of ethyl acetate and ethyl ether with volume ratio $1: 1$, the organic phases were combined. The organic layer was dried by anhydrous sodium sulfate and concentrated at $50{ }^{\circ} \mathrm{C}$ using rotary evaporator until organic phase was evaporated to dryness. Finally, the residue was diluted with $2 \mathrm{~mL}$ acetonitrile and the solution was filtered with $0.22 \mu$ m nylon membrane.

Instruments and chromatographic conditions: The fresh tobacco were dried by a PiloFD-4.3V freeze dryer (SIM International Group Co. Ltd., Newark, USA). The centrifugation was carried out using a 5804R refrigerated centrifuge (Eppendorf, Hamburg, Germany). Phenolic acids was analyzed by HPLC (Waters Corporation, Milford, MA, USA) equipped with a $\mathrm{C}_{18} \mathrm{ODS}_{2}$ column $(4.6 \mathrm{~mm} \times 250 \mathrm{~mm}, 5 \mu \mathrm{m}$; waters corporation, ireland). The mobile phases were $2 \%$ acetic acid aqueous solution (A) and $2 \%$ acetic acid acetonitrile solution (B). The program was as follows: linear gradient from $95 \%$ $\mathrm{A} / 5 \% \mathrm{~B}$ to $70 \% \mathrm{~A} / 30 \% \mathrm{~B}, 0-40 \mathrm{~min}$; linear gradient from $70 \% \mathrm{~A} / 30 \% \mathrm{~B}$ to $95 \% \mathrm{~A} / 5 \% \mathrm{~B}, 40-50$ min. The column temperature was $35^{\circ} \mathrm{C}$ and the flow rate was $1.0 \mathrm{~mL} \mathrm{~min}^{-1}$. The detection wavelength was $310 \mathrm{~nm}$ and the injection volume was $4 \mu \mathrm{L}$.

The C6-C3 phenolic acid compounds were qualitatively detected by the comparisons of the reference samples in terms of chromatographic retention time and it was quantitatively detected by the calibration curves with the internal standard methods.

\section{RESULTS AND DISCUSSION}

Optimization of wavelength: In order to obtain the maximum absorption wavelength of each compound, the full wavelength was scanned at $190-400 \mathrm{~nm}$. The results showed that there were two maximum absorption regions which existed in the range of 230-235 and 320-330 nm. $310 \mathrm{~nm}$ was selected as the detection wavelength due to the high degree of separation and signal-to-noise ratio obtained.

Optimization of extraction solvents: Both free and bound phenolic acid compounds in tobacco samples were analyzed. The free C6-C3 phenolic acid compounds were extracted with different concentration of methanol. The results showed that the free C6-C3 phenolic acid compounds wasn't detected in the solution extracted by $70 \%$ methanol, $80 \%$ methanol solution and $100 \%$ methanol for $0.5,1.0$ and $2.0 \mathrm{~h}$, respectively and the result was also validated with UPLC-Xevo QTof MS. Since there is no free phenolic acid was found in tobacco, we mainly focus on the method of bound phenolic acid in the following study.

Based on previous studies of bound phenolic acids in other crops $^{9,15,20}, 1,2$ and $4 \mathrm{~mol} / \mathrm{L} \mathrm{NaOH}$ solutions were used as extraction solvents. As shown in Table-2, the phenolic acid contents were highest in $4 \mathrm{~mol} / \mathrm{L} \mathrm{NaOH}$ solution and $4 \mathrm{~mol} / \mathrm{L}$ $\mathrm{NaOH}$ was further applied to analysis of tobacco samples.

\begin{tabular}{cccc}
\multicolumn{4}{c}{ TABLE-2 } \\
\multicolumn{4}{c}{ CONTENT OF BOUND C6-C3 PHENOLIC } \\
ACIDS IN TOBACCO WITH DIFFERENT \\
EXTRACTION SOLVENTS $(\mu \mathrm{g} / \mathrm{g})$ \\
\hline Compounds & $\mathrm{NaOH}$ & $\mathrm{NaOH}$ & $\mathrm{NaOH}$ \\
& $(1 \mathrm{~mol} / \mathrm{L})$ & $(2 \mathrm{~mol} / \mathrm{L})$ & $(4 \mathrm{~mol} / \mathrm{L})$ \\
\hline Caffeic acid & 1.15 & 1.23 & 1.24 \\
$\mathrm{p}-$ Coumaric acid & 22.03 & 24.08 & 24.76 \\
Ferulic acid & 10.56 & 12.76 & 12.98 \\
Sinapic acid & 7.15 & 8.54 & 9.03 \\
\hline
\end{tabular}

In present study, free $\mathrm{C} 6-\mathrm{C} 3$ phenolic acids weren't detected in tobacco leaves, but the bound C6-C3 phenolic acids could be released under strong alkali condition. In order to prevent ionization of carboxyl and phenolic hydroxyl groups the solutions A ( $2 \%$ acetic acid solution)-B ( $2 \%$ acetic acid acetonitrile solution) were selected as mobile phase to achieve the four kinds of C6-C3 phenolic acids well separated within $40 \mathrm{~min}$. In previous studies, caffeic acid, $p$-coumaric acid, ferulic acid and sinapic acid were separated at 31-80 min with RP-C18 (LiChroCART, $250 \times 4 \mathrm{~mm}, 5 \mu \mathrm{m}$ ) for separation and solvent $\mathrm{A}(0.02 \mathrm{M}$ phosphate buper, $\mathrm{pH} 2.15)$ and solvent B (methanol: 0. 02M phosphate buper, pH 2.15, 40:60 v/v) for elution ${ }^{17}$ and caffeic acid, $p$-coumaric acid and ferulic acid were separated at 57 min using C18 (Phenomenex, $150 \times 4.6$ $\mathrm{mm} ; 5 \mu \mathrm{m}$ ) with mobile phase in (A) $0.1 \%$ formic acid and (B) methanol ${ }^{9}$. Though caffeic acid and $p$-coumaric acid were separated at 20-28 min with using inertsil C18 ODS-3 (150 $\times$ $4.0 \mathrm{~mm}, 3 \mu \mathrm{m}$; GL Sciences, Inc., Japan) and mobile phase was consisted of $50 \mathrm{mM} \mathrm{H}_{3} \mathrm{PO}_{4}$, pH 2.5 (A) and acetonitrile (B), however, peaks of ferulic acid and sinapic acid were completely overlaid together ${ }^{7}$. Compare with previous studies ${ }^{7,9,17,20}$, it was reduced the separation time and separated well of the four C6-C3 phenolic acids in our research.

Meanwhile, the extraction conditions in light of the major features of C6-C3 phenolic acids in tobacco were optimized. During the experimental process, it was found that the accuracy and precise could be improved, when the two problems of 
filtering blockage and emulsification in extraction were processed with the low-temperature high-speed centrifugation.

Linearity and limit of detection: The linearity of the method was evaluated by the regression equation of C6-C3 phenolic acid with the concentration of the reference substance as the X-coordinate and the peak area/internal standard peak area as Y-coordinate (Table-3). The results showed that a good linearity can be found when the concentration in the range of $1.113-102.332 \mu \mathrm{g} / \mathrm{mL}$ with all correlation coefficient higher or equal to 0.9992 . The limit of detection was calculated with signal-to-noise ratio $\mathrm{S} / \mathrm{N}=3$.

\begin{tabular}{ccccc}
\multicolumn{6}{c}{ TABLE-3 } \\
REGRESSION EQUATIONS OF C6-C3 PHENOLIC ACIDS \\
\hline Compounds & Regression equation & $\mathrm{R}^{2}$ & $\begin{array}{c}\text { Concentration } \\
\text { range }(\mu \mathrm{g} / \mathrm{mL})\end{array}$ & $\begin{array}{c}\text { Limit of } \\
\text { detection } \\
(\mathrm{ng} / \mathrm{mL})\end{array}$ \\
\hline $\begin{array}{c}\text { Caffeic acid } \\
\text {-Coumaric acid }\end{array}$ & $\mathrm{y}=0.033 \mathrm{x}=0.056 \mathrm{x}-0.0165$ & 0.9994 & $1.113-56.236$ & 21.4 \\
Ferulic acid & $\mathrm{y}=0.0327 \mathrm{x}-0.0064$ & 0.9995 & $1.110-56.620$ & 30.4 \\
Sinapic acid & $\mathrm{y}=0.0211 \mathrm{x}-0.0167$ & 0.9992 & $1.067-53.881$ & 25.5 \\
\hline
\end{tabular}

Repeatability and stability test: The repeatability of proposed methods was evaluated by using 6 replicates for the same batch. The relative standard deviation (RSD) of bound phenolic acids including caffeic acid, $p$-coumaric acid, ferulic acid and sinapic acid were $3.21,4.76,3.73$ and $4.52 \%$, respectively. Furthermore, the same samples were reanalyzed for the stability at $0,2,4,8,12$ and $24 \mathrm{~h}$ after extracted respectively and the RSD of caffeic acid, $p$-coumaric acid, ferulic acid and sinapic acid were $0.16,0.31,0.42$ and $0.35 \%$, respectively. The results demonstrated that the proposed method had high repeatability and stability. A typical standard and sample chromatogram were shown in Fig. 1.

Recovery: Accurately $0.6000 \mathrm{mg}$ of powder tobacco samples was weighed and mixed with reference substances caffeic acid, $p$-coumaric acid, ferulic acid and sinapic acid according to the experimental method in above, respectively. Then, the recovery was calculated according to the amount of standard samples and the quantity of samples before and after the addition of reference substances. The results showed that the recovery was $97.12,97.32,96.16$ and $96.73 \%$, respectively.

Applications: The tobacco leaves from five provinces of China (Heilongjiang, Shandong, Shanxi, Guizhou and Chongqing) in 2013 were sampled at different growth stages and the phenolic acids were determined with newly established method. The results showed that the content of caffeic acid in fresh tobacco was very low (Table-4) and caffeic acid was only detected in samples from Heilongjiang at 10 days and 20 days after flowering and samples from Chongqing at 10 days after flowering, with the content ranged from 0.44 to $2.07 \mu \mathrm{g} / \mathrm{g}$. In the other hand, analysis of variance (Table-5) showed that except caffeic acid the influence of region, growth stage and their interaction on contents of $p$-coumaric acid, ferulic acid and sinapic acids were highly significant.

Our study indicated that the contents of $p$-coumaric acid, ferulic acid and sinapic acid in flue-cured tobacco leaves had significant differences among different regions and growth stages. These results were consistent with previous report in which different growth stages and environments could influence the quantity and types of the metabolism in cell ${ }^{21}$.

\section{Conclusion}

Considering overall experiment costs, separation time, accuracy and stability, the method which was consisted of $4 \mathrm{~mol} / \mathrm{L} \mathrm{NaOH}$ as extraction solvent and A (2\% acetic acid solution)-B ( $2 \%$ acetic acid in acetonitrile solution) as mobile phase was optimized to separate caffeic acid, $p$-coumaric acid, ferulic acid and sinapic acid. Its high sensitivity, accuracy and repeatability could be employed to determination of contents of C6-C3 phenolic acids in flue-cured and fresh tobacco.
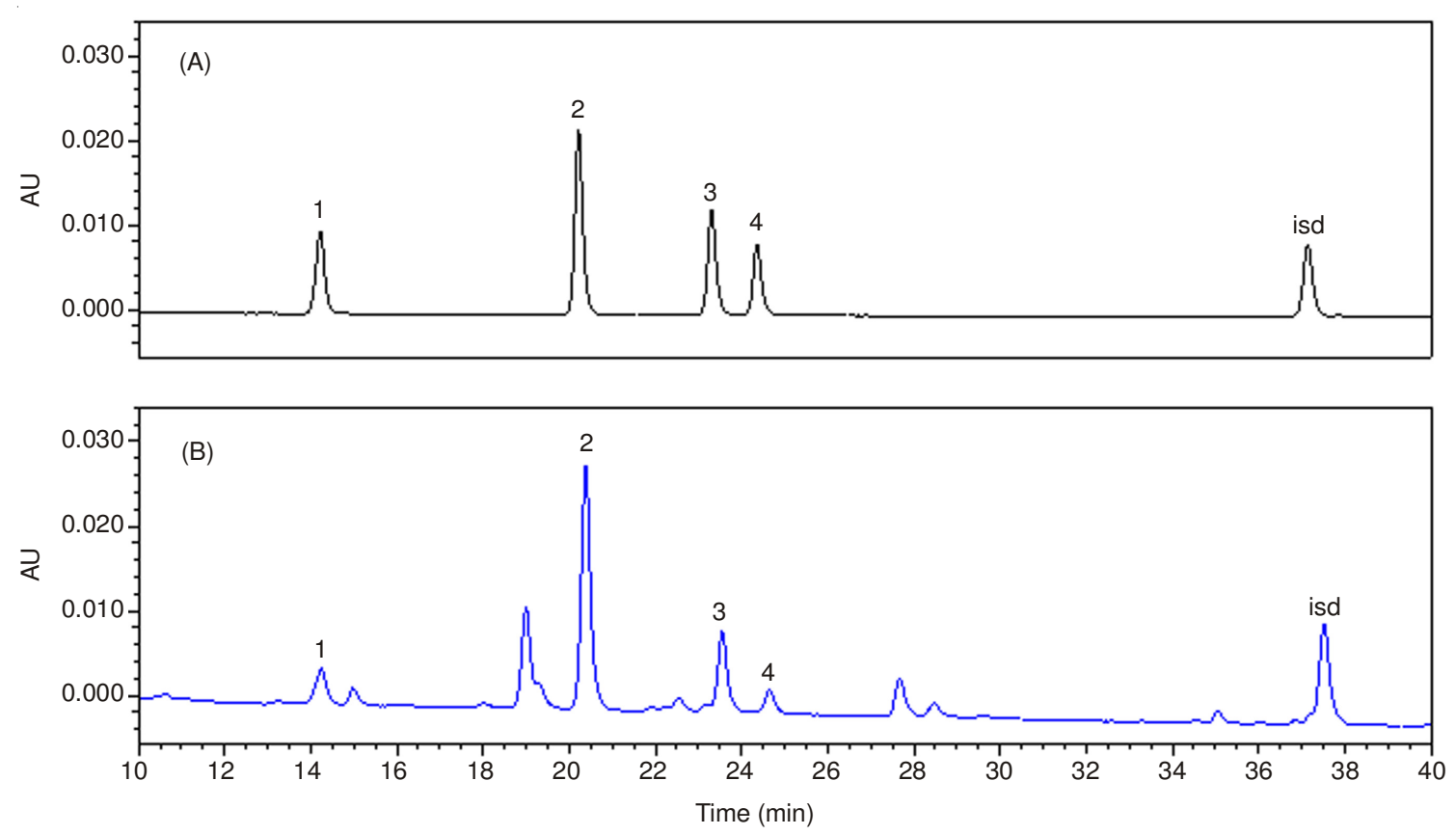

Fig. 1. HPLC chromatogram of standard samples (A) and tobacco samples (B) (1) caffeic acid; (2) p-coumaric acid; (3) ferulic acid; (4) sinapic acid; isd:internal standard (cinnamic acid) 
TABLE-4

CONTENT OF BOUND C6-C3 PHENOLIC ACIDS IN TOBACCO AT DIFFERENT GROWTH STAGES (DRY WEIGHT, $\mu \mathrm{g} / \mathrm{g}$ )

\begin{tabular}{cccccc}
\hline Regions & Growth stage & Caffeic acid & $p$-Coumaric acid & Ferulic acid & Sinapic acid \\
\hline \multirow{5}{*}{ Chongqing } & 10 Days after flowering & $1.44 \pm 0.23$ & $41.46 \pm 4.44$ & $14.82 \pm 5.10$ & $9.77 \pm 2.24$ \\
& 20 Days after flowering & $\mathrm{N}$ & $43.68 \pm 8.77$ & $15.49 \pm 2.44$ & $12.56 \pm 2.02$ \\
& 30 Days after flowering & $\mathrm{N}$ & $29.44 \pm 2.15$ & $16.86 \pm 1.95$ & $13.31 \pm 1.36$ \\
& 1 Day before harvest & $\mathrm{N}$ & $32.25 \pm 5.75$ & $14.81 \pm 4.49$ & $17.08 \pm 3.74$ \\
\hline \multirow{5}{*}{ Heilongjiang } & 10 Days after flowering & $1.02 \pm 0.02$ & $93.80 \pm 16.37$ & $22.95 \pm 6.42$ & $12.80 \pm 2.53$ \\
& 20 Days after flowering & $2.07 \pm 0.93$ & $26.98 \pm 3.56$ & $25.25 \pm 5.36$ & $12.74 \pm 4.05$ \\
& 30 Days after flowering & $\mathrm{N}$ & $19.83 \pm 2.94$ & $18.34 \pm 6.96$ & $24.49 \pm 4.07$ \\
& 40 Days after flowering & $\mathrm{N}$ & $20.22 \pm 1.56$ & $14.13 \pm 3.18$ & $33.50 \pm 5.95$ \\
& 1 Day before harvest & $\mathrm{N}$ & $19.24 \pm 2.34$ & $17.87 \pm 2.59$ & $37.76 \pm 6.63$ \\
\hline \multirow{5}{*}{ Shandong } & 10 Days after flowering & $\mathrm{N}$ & $26.31 \pm 3.24$ & $22.85 \pm 5.65$ & $6.59 \pm 1.33$ \\
& 20 Days after flowering & $\mathrm{N}$ & $30.77 \pm 3.90$ & $14.96 \pm 2.14$ & $6.09 \pm 0.61$ \\
& 30 Days after flowering & $\mathrm{N}$ & $20.31 \pm 1.28$ & $16.13 \pm 1.13$ & $6.62 \pm 0.26$ \\
& 40 Days after flowering & $\mathrm{N}$ & $16.22 \pm 0.98$ & $11.55 \pm 0.94$ & $15.34 \pm 1.71$ \\
\hline \multirow{5}{*}{ Shanxi } & 1 Day before harvest & $\mathrm{N}$ & $15.21 \pm 3.60$ & $16.03 \pm 5.77$ & $12.54 \pm 2.43$ \\
& 10 Days after flowering & $\mathrm{N}$ & $63.26 \pm 6.50$ & $20.08 \pm 4.44$ & $11.09 \pm 1.99$ \\
& 20 Days after flowering & $\mathrm{N}$ & $45.39 \pm 5.91$ & $14.44 \pm 5.23$ & $13.89 \pm 4.52$ \\
& 30 Days after flowering & $\mathrm{N}$ & $59.76 \pm 8.76$ & $30.72 \pm 14.19$ & $23.68 \pm 5.48$ \\
& 40 Days after flowering & $\mathrm{N}$ & $45.87 \pm 4.75$ & $24.76 \pm 1.31$ & $21.62 \pm 5.34$ \\
Guizhou & 1 Day before harvest & $\mathrm{N}$ & $45.91 \pm 4.73$ & $24.78 \pm 1.25$ & $21.64 \pm 5.40$ \\
\hline & 10 Days after flowering & $\mathrm{N}$ & $57.96 \pm 5.01$ & $13.01 \pm 2.42$ & $5.98 \pm 1.00$ \\
& 20 Days after flowering & $\mathrm{N}$ & $52.39 \pm 5.81$ & $20.93 \pm 1.80$ & $7.74 \pm 0.85$ \\
& 30 Days after flowering & $\mathrm{N}$ & $41.15 \pm 3.25$ & $15.77 \pm 2.66$ & $14.48 \pm 1.96$ \\
\hline N means not detected & 1 Day before harvest & $\mathrm{N}$ & $45.93 \pm 6.56$ & $18.36 \pm 1.45$ & $29.91 \pm 3.55$ \\
\hline
\end{tabular}

TABLE-5

VARIANCE ANALYSIS OF BOUND C6-C3 PHENOLIC ACIDS IN TOBACCO AT DIFFERENT GROWTH STAGES AND REGIONS

\begin{tabular}{|c|c|c|c|c|c|c|}
\hline Origin & Dependent variable & Sum of squares & Degree of freedom & Mean square & $\mathrm{F}$ & Significance \\
\hline \multirow{3}{*}{ Region } & $p$-Coumaric acid & 16178.48 & 4 & 4044.62 & 118.68 & 0.000 \\
\hline & Ferulic acid & 1178.22 & 4 & 294.55 & 12.71 & 0.000 \\
\hline & Sinapic acid & 3475.90 & 4 & 868.98 & 70.15 & 0.000 \\
\hline \multirow{3}{*}{ Growth stage } & $p$-Coumaric acid & 13189.29 & 4 & 3297.32 & 96.75 & 0.000 \\
\hline & Ferulic acid & 214.65 & 4 & 53.66 & 2.32 & 0.000 \\
\hline & Sinapic acid & 4801.06 & 4 & 1200.27 & 96.89 & 0.000 \\
\hline \multirow{3}{*}{ Region $\times$ growth stage } & $p$-Coumaric acid & 16826.24 & 14 & 1201.87 & 35.27 & 0.000 \\
\hline & Ferulic acid & 1766.04 & 14 & 126.15 & 5.45 & 0.000 \\
\hline & Sinapic acid & 1851.28 & 14 & 132.23 & 10.67 & 0.000 \\
\hline
\end{tabular}

\section{ACKNOWLEDGEMENTS}

This work was supported by grants from the Key Special Program of China National Tobacco Corporation (TS-0220110014), the Foundation of Science and Technology of Guizhou Province of China (J[2013]2195) and the Program of Guizhou Provincial Tobacco Company (201431).

\section{REFERENCES}

1. E. Anouar, P. Košinová, D. Kozlowski, R. Mokrini, J.L. Duroux and P. Trouillas, Chem. Chem. Phys., 11, 7659 (2009).

2. $\quad$ S.B. Revanappa and P.V. Salimath, J. Food Biochem., 35, 759 (2011).

3. K.H. Kim, R. Tsao, R. Yang and S.W. Cui, Food Chem., 95, 466 (2006).

4. U. Gawlik-Dziki, M. Swieca and D. Dziki, J. Agric. Food Chem., 60, 4603 (2012)

5. A. Caniova and E. Brandsteterova, Chem. Anal. (Warsaw), 46, 757 (2001).

6. K. Aaby, D. Ekeberg and G. Skrede, J. Agric. Food Chem., 55, 4395 (2007).

7. P. Mattila and J. Kumpulainen, J. Agric. Food Chem., 50, 3660 (2002).

8. C. del Mar Verde Méndez, M.Á. Rodríguez Delgado, E.M. Rodríguez Rodríguez and C. Díaz Romero, J. Agric. Food Chem., 52, 1323 (2004).
9. D.L. Luthria, S. Mukhopadhyay and D. Krizek, J. Food Compos. Anal., 19, 771 (2006).

10. B. Dimitrova, R. Gevrenova and E. Anklam, Phytochem. Anal., 18, 24 (2007).

11. K. Pyrzynska and M. Biesaga, Trends Analyt. Chem., 28, 893 (2009).

12. J. Lachman, A. Hejtmánkova, J. Sykora, J. Karban, M. Orsák and B. Rygerová, Czech J. Food Sci., 28, 412 (2010).

13. S. Qiao, R. Shi, M. Liu, C. Zhang, W. Yang, X. Shi, X. Jiang, C. Wang and Q. Wang, Food Chem., 129, 1297 (2011).

14. Z.C. Zhang, M. Xu, S.F. Sun, X. Qiao, B.R. Wang, J. Han and D.A. Guo, J. Chromatogr. B Analyt. Technol. Biomed. Life Sci., 871, 7 (2008).

15. A. Olkowski, R. Amarowicz, P. Yu, J.J. McKinnon and D.D. Maenz, Pol. J. Food Nutr. Sci., 53, 53 (2003).

16. M. Nikolova and R. Gevrenova, Pharm. Biol., 43, 289 (2005).

17. P.A. Kroon and G. Williamson, Biotechnol. Appl. Biochem., 23, 263 (1996).

18. R. Amarowicz and P.P. Kolodziejczyk, Nahrung, 45, 62 (2001).

19. M.F. Andreasen, L.P. Christensen, A.S. Meyer and Hansen, J. Sci. Food Agric., 79, 411 (1999).

20. L. Barros, M. Dueñas, I.C.F.R. Ferreira, P. Baptista and C. SantosBuelga, Food Chem. Toxicol., 47, 1076 (2009).

21. K. Saito and F. Matsuda, Annu. Rev. Plant Biol., 61, 463 (2010). 